\title{
Editorial
}

\section{Screening for Ovarian Cancer}

Ovarian cancer accounts for about $3 \%$ of cancers in women, but it is the leading cause of death from gynecologic cancers and the fifth leading cause of all deaths related to cancer among women. ${ }^{1}$ In the United States, the lifetime risk of invasive ovarian cancer is approximately $1.4 \%$ ( 1 in 71 ), and the lifetime risk of dying from invasive ovarian cancer is about 1 in $95 .{ }^{1}$ In India $4.9 \%$ of cancers in female were ovarian cancer. ${ }^{2}$

Two thirds of ovarian cancer are diagnosed in women over the age of 55 years. ${ }^{2,3} \mathrm{~A}$ family history of ovarian or breast cancer in a first-degree relative approximately triples the risk. ${ }^{3}$ The risk is high among carriers of a BRCA gene mutation. The risk is decreased among women who have been pregnant, have history of breast-feeding or have used oral contraceptives. ${ }^{3}$

More than two thirds of ovarian cancer are diagnosed when the disease has progressed to stage III or IV and involves the peritoneal cavity or other organs. ${ }^{4}$ Symptoms that are associated with ovarian cancer are typically nonspecific, and the association is often not recognized until the disease has advanced. ${ }^{5}$ Current treatment includes surgical resection (debulking), followed by multiagent chemotherapy, usually involving intravenous or intraperitonealplatin compounds and a taxane. Prognostic factors are: the stage and histologic grade of the cancer at diagnosis, the presence or absence of residual disease at the completion of the initial surgery, the patient's functional status and age, and the use or nonuse of platinbased chemotherapy. The 5-year survival rate is approximately $90 \%$ when ovarian cancer is detected and treated while it is still confined to the ovary (stage I), in contrast to the rate of approximately $33 \%$ when the disease is diagnosed at stage III or IV. ${ }^{4}$ Since ovarian cancer is often initially diagnosed at an advanced stage, when the prognosis is poor even with aggressive therapy, a screening method that facilitates early diagnosis has been actively sought. Routine Screening Criteria for disease screening have been proposed by the World Health Organization. ${ }^{5}$ Some of these criteria are met for ovarian cancer, since the condition is frequently fatal, treatment is available that is effective at an early stage of disease, and early intervention improves the outcome. However, several features of ovarian cancer complicate the question of screening. First, the transition time from stage I to stage III is unclear, since it is not known whether there is an evolution from an early to an advanced stage or whether the disease may initially arise as a diffuse process in the peritoneal cavity (stage III). Furthermore, because there is no obvious precursor lesion, screening must focus on early detection of invasive cancer. Risk factors other than age, a family history of ovarian or breast cancer, and the presence of a BRCA mutation are poorly understood, and approximately $90 \%$ of ovarian cancers appear to be sporadic. Thus, screening algorithms must be geared toward a general population of women.

As only a small fraction of cases of ovarian cancer occur in premenopausal women, most investigators have suggested that screening be limited to postmenopausal women. Moreover, because of the low prevalence of ovarian cancer (40 cases per year per 100,000 women over the age of 50 years), a screening test must have both high sensitivity and high specificity to be clinically useful.

It is estimated that a screening test for ovarian cancer would require a sensitivity of at least $75 \%$ and a specificity of more than $99.6 \%$ to achieve a positive predictive value of $10 \%$. Since a definitive diagnosis of ovarian cancer requires surgical excision of the ovary and fallopian tube, a screening test with a positive predictive value of $10 \%$ would result in 10 surgeries for every 1 case of cancer detected. Currently available screening tests for ovarian cancer must be considered in light of these issues. ${ }^{6}$

The current recommendations against screening for ovarian cancer are based on the large U.S. prospective randomized Prostate, Lung, Colorectal and Ovarian (PLCO) Cancer Screening Trial. ${ }^{7}$ The PLCO trial demonstrated that an annual cancer antigen (CA) 125 measurement (using a fixed cutoff value for a positive test result) and ultrasonography were not associated with a reduction in mortality from ovarian cancer. Furthermore, screening was associated with significant harms resulting from surgeries that were triggered by false-positive findings.

In December 2015, the results of the UKCTOCS trial were reported in The Lancet. ${ }^{8}$ This landmark study included approximately 200,000 healthy 
postmenopausal women, of whom one-half were randomized to no screening, one-fourth were randomized to receive annual pelvic ultrasonography, and one-fourth were randomized to multimodal screening (MMS). In contrast to the PLCO approach, MMS involves a risk of ovarian cancer algorithm $(\text { ROCA })^{9}$ that assigns a level of risk based on an individual woman's CA 125 levels and changes over time combined with her age and known risk factors for ovarian cancer. Based on these findings, further CA 125 testing and ultrasonography may be required.The primary analysis suggested a nonsignificant mortality reduction over years 0 to 14 of $15 \%$ in the MMS arm vs. the usual care arm. However, the reduction in mortality was not constant over time, appearing only after seven to 10 years of screening. Compared with annual, fixed-cutoff CA 125 levels and ultrasonography, as were studied in the PLCO trial, the MMS algorithm was more sensitive and led to fewer unnecessary surgeries. The UKCTOCS trial demonstrated a stage shift in which more cases were diagnosed at an early stage with the MMS approach (40\%) compared with women in the nonscreened (usual care) group (26\%). There was no stage shift observed for the group receiving ultrasonography alone. Additional exploratory analysis suggested even more encouraging, though as yet inconclusive, evidence of the potential for a greater mortality reduction in the MMS group.

As stated in The Lancet UKCTOCS paper, "further follow-up is needed before firm conclusions can be reached on the efficacy and cost-effectiveness of ovarian cancer screening". 8 In February 2016, the Ovarian Cancer Research Fund Alliance convened a group of 25 scientists, clinicians, and advocates to meet at the Banbury Center, to discuss the recent results from the United Kingdom Collaborative Trial of Ovarian Cancer Screening (UKCTOCS) and implications for clinical practice and public health. ${ }^{10}$ The consensus of the Banbury group is in accord with the published conclusions of the UKCTOCS trial: It is premature to recommend MMS for the early detection of ovarian cancer at this time. Although women and health care professionals may look to these findings as finally providing a strategy for successful screening for ovarian cancer, the study investigators and participants at this meeting believe that screening policy requires a sound scientific foundation, which we currently do not have. The study results will be reanalyzed using the additional data in three years, and firmer conclusions may emerge. This analysis will be eagerly awaited, because there will not be a similarly powered ovarian cancer screening study in the foreseeable future.

The (ROCA), a component of the MMS strategy evaluated in the UKCTOCS trial, is already commercially available. Although no organization recommends ovarian cancer screening in averagerisk women, some women may wish to undergo periodic screening for ovarian cancer with CA 125 testing; therefore, health care professionals must advise women regarding the potential benefits and risks as we understand them now.

\section{Summary of the recommendationand suggestions for clinical practice ${ }^{11}$.}

Population Asymptomatic women without known genetic mutations that increase risk for ovarian cancer

Recommendation

Risk Assessment

Screening Tests

Treatments

Balance of Benefits and Harms

\section{Do not screen for ovarian cancer. Grade: D}

Women with BRCA1 and BRCA2 genetic history of ovarian cancer are at increased risk for ovarian cancer.Women with an increased mutations, the Lynch syndrome (hereditary nonpolyposis colon cancer), or an 'increased-risk family history' should be considered for genetic counseling to further evaluate their potentialrisks. "Increasedrisk family history" generally means having 2 or more first- or second-degree relatives with a history ofovarian cancer or a combination of breast and ovarian cancer; for women of Ashkenazi Jewish descent, it means having afirst-degree relative (or 2 second-degree relatives on the same side of the family) with breast or ovarian cancer.

Transvaginal ultrasonography and serum cancer antigen (CA)-125 testing are the most commonly suggested screening tests.

Treatment of ovarian carcinoma includes surgical treatment (debulking) and intraperitoneal or systemic chemotherapy.

Annual screening with transvaginal ultrasonography and serum CA-125 testing in women does not decrease ovarian cancer mortality. Screening for ovarian cancer can lead to important harms, including major surgical interventions in women who do not have cancer. Therefore, the harms of screening for ovarian cancer outweigh the benefits. 
Consensus among major medical and public health organizations is that screening for ovarian cancer in the general population is not recommended. The American Congress of Obstetricians and Gynecologists does not recommend screening for ovarian cancer in asymptomatic women; evaluation of high-risk persons may include Transvaginal ultrasonography and CA-125 testing in addition to physical examination. ${ }^{12}$ The American Cancer Society states that no screening test has proven to be effective and sufficiently accurate for early detection of ovarian cancer. However, for women who are at high risk, the combination of a thorough pelvic examination, transvaginal ultrasonography, and a blood test for the tumor marker CA- 25 may be offered. ${ }^{13}$

\section{Prof. Ferdousi Begum \\ Editor}

\section{References}

1. Jemal A, Siegel R, Ward E, et al. Cancer statistics, 2008. CA Cancer J Clin 2008; 58: 71-96.

2. Rastogi T, DevesaS, Mangtani P, Mathew A, Cooper N, Kao R and Sinha R. Cancer incidence rates among South Asians in four geographic regions: India, Singapore, UK and US. International Journal of Epidemiology 2008;37:147-160

3. Whittemore AS. Characteristics relating to ovarian cancer risk: implications for prevention and detection. GynecolOncol 1994;55:S15-S19.

4. Heintz APM, Odicino F, Maisonneuve P, et al. Carcinoma of the ovary. Int J Gynaecol Obstet 2006;95:Suppl 1:S161-S192.

5. Wilson JMG, Junger G. Principles and practice of screening for disease. Geneva: World Health Organization, 1968.
6. Rosenthal AN, Menon U, Jacobs IJ. Screening for ovarian cancer.ClinObstetGynecol 2006; 49:433.

7. Buys SS, Partridge E, Black A, et al.; PLCO Project Team. Effect of screening on ovarian cancer mortality: the Prostate, Lung, Colorectal and Ovarian (PLCO) Cancer Screening Randomized Controlled Trial. JAMA. 2011;305(22):2295-2303.

8. Jacobs IJ, Menon U, Ryan A, et al. Ovarian cancer screening and mortality in the UK Collaborative Trial of Ovarian Cancer Screening (UKCTOCS): a randomised controlled trial Lancet. 2016;387(10022):945-956.

9. Skates SJ. OCS: Development of Risk of Ovarian Cancer Algorithm (ROCA) and ROCA screening trials. Int J Gynecol Cancer. 2012May; 22(Suppl1): S24-S26.

10. Ovarian Cancer Research Fund Alliance and Banbury Conference Writing Group. What Women and Their Physicians Need to Know About the UKCTOCS Study and Ovarian Cancer Screening. Am Fam Physician. 2016 Jun 1; 93(11): 903-904.

11. Virginia A, on behalf of the U.S. Preventive Services Task Force. Screening for Ovarian Cancer: U.S. Preventive Services Task Force Reaffirmation Recommendation Statement. Ann Intern Med. 2012;157:900-904.

12. American College of Obstetricians and Gynecologists Committee on Gynecologic Practice. Committee Opinion No. 477: the role of the obstetriciangynecologistin the early detection of epithelial ovarian cancer. Obstet Gynecol. 2011;117:742-6. [PMID: 21343791]

13. American Cancer Society. Cancer Facts \& Figure 2012. Atlanta: AmericanCancerSoc; 2012. Accessed at www.cancer.org/Research/ CancerFactsFigures/CancerFactsFigures/ cancer-facts-figures-2012 on 2 April 2012. 